\title{
Comparison of a 3-D Expression System and a Standardized IQ Test for Children
}

\author{
Akihiro Suzuki, Masayuki Wajima, Takashi Kawakami, and Tetsuo Okazaki \\ 7-15 Maeda, Teine-ku, Sapporo, Hokkaido, Japan \\ \{r09301,wajima, kawakami, okazaki\}@hit.ac.jp
}

\begin{abstract}
The objective of this paper is to analyze how children develop their spatial expression ability in 3-D space. We prepared a 3-D paint system that has "Translation-test", "Rotation-test" and "Paper-lay-out-test". As an experiment, we collected data by using our 3-D paint system for children between the ages of 4 and 6 in a pre-school in August 2009, January 2010, and August 2010. We conducted a survey on correlation between the system and WPPSI: WPPSI is an IQ test. At these experiments, we conducted 5 performance tests with WPPSI. The results are summarized as follows: 1) Children are able to recognize virtual 3-D space of the system, and are able to express images in virtual 3-D space; 2) The data shows the trend that spatial expression ability in space becomes better with advancing ages; 3 ) We obtained correlations between the system and WPPSI. The system can analyze some abilities that are difficult for WPPSI.
\end{abstract}

Keywords: Spatial vision, Spatial expression, Human-computer-interaction, Children.

\section{Introduction}

Various articles have been published on the process of how children in Japan develop their spatial perception. For example, these articles try to understand it by means of studying paintings[1], using toy blocks[2] and having conversations[3][4]. However, paintings depends on 2-D expression. And with toy blocks, children are highly likely to have space with different parameters. Children likely to have a different sense of gravity and children's space likely to have a different coordinate axis from our adult's space. And children's conversational ability needs a long period for development. The Wechsler Preschool and Primary Scale of Intelligence test (WPPSI)[5], one of the standardized IQ tests, has subtests to analyze spatial perception ability. But the WPPSI analyzes ability using 2-D space. Moreover, test examiners need to understand the WPPSI test, and children need to endure a test period of longer than 2 hours.

So, we feel that our approach, which uses a computer, is more effective. We prepared a 3-D paint system to measure the developmental growth of spatial expression ability in 3-D space. As far as we know, this is the first approach that studies children's spatial development using a computer. We conducted a series of experiments with 4 to 6 years old children in a pre-school, as the first step in studying 
the development of spatial growth. The aim of the experiment is to measure how children develop their spatial expression ability over a period of time.

\section{Prepared System}

\subsection{3-D Paint System}

We define some basic spatial expression abilities as follows:

- Operation abilities in defined 3-D space

- ability to cognize and understand a limited 3 dimensional area

- ability to move something to a target position in a space, or ability to draw a figure in a 3 dimensional area

- Expression abilities of relations

- ability to cognize relations of distances, directions, and dimensions between one thing and the other

- ability to decide distances, directions, and dimensions for the target relations

Our 3-D paint system needs functions for understanding those abilities, and needs methods of spatial expressions for understanding that children's immanent 3-D space. So, we formulated the following methods of spatial expressions in a computer, and employed those methods on our 3-D paint system. Fig.1 is a mock picture showing the stage of expression of complex perspective (4 to 7 years old) according to Higashiyama[1]. The picture has a road and a railway track from an overhead view and a house and a train from lateral view. A 3-D image can be expressed by dividing Fig.1 into various parts and to lay out the parts in a 3-D image as Fig.2. Similarly, we applied this method to the system. The system has a function that a user to lay out illustrated papers in a virtual 3-D program. We also equipped the system with other functions by means of operating cubes for understanding the operation abilities.

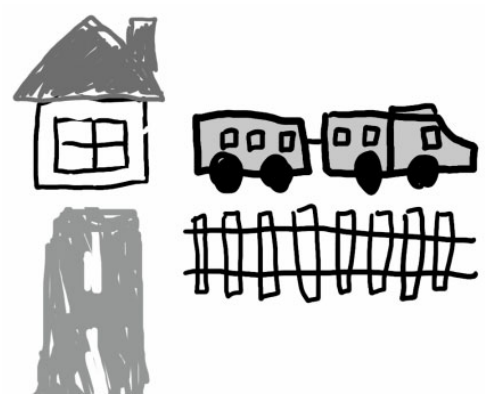

Fig. 1. A child's drawing

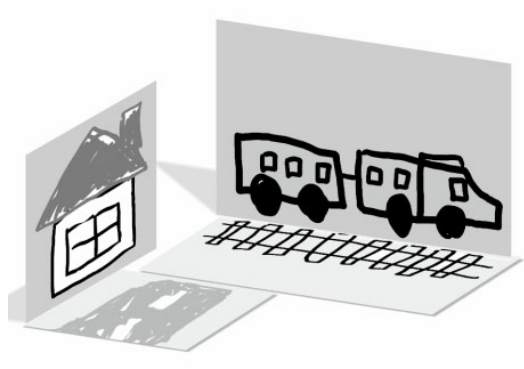

Fig. 2. Lay out in 3-D space 


\subsection{Overview of the System}

We prepared a 3-D software paint system which meets the requirements in section 2.1. We developed the system using the $\mathrm{C}++$ programming language, the system works on Windows and Mac OS X. Fig.3 shows the execution screen of the system. The function buttons are located at the top of the screen. Located at the left of the screen are a color palette, undo button and redo button, and the button that can be used to create a paper. The rest of the area is space to express 3-D images.

We applied Nintendo's Wii Remote[6] as an operation device for the system. Because, to use a mouse and a manipulator, which are commonly used in computer graphics, is hard for children who have limited or no computer experiences. The operation device for the system requires acceleration sensors and gyroscope sensors to rotate the papers, and optical sensors and buttons to translate and choose the papers. Applying Wii Remote to the system is useful in introducing the system at a pre-school or at home. Because anyone can obtain Wii Remote easily. So, we adopted Wii Remote for the system. We henceforth call Wii Remote "3-D Remote".

The system has functions of creating new papers, drawing on papers, displaying cubes for experiments, translating and rotating papers and cubes, and translating and rotating a view. And the system has functions of Translation-test, Rotation-test, Paper-lay-out-test for experiments. The Translation-test and the Rotation-test are tests for analyzing the operation abilities in 3-D space. The Paper-lay-out-test is a test for analyzing the expression abilities of relations.

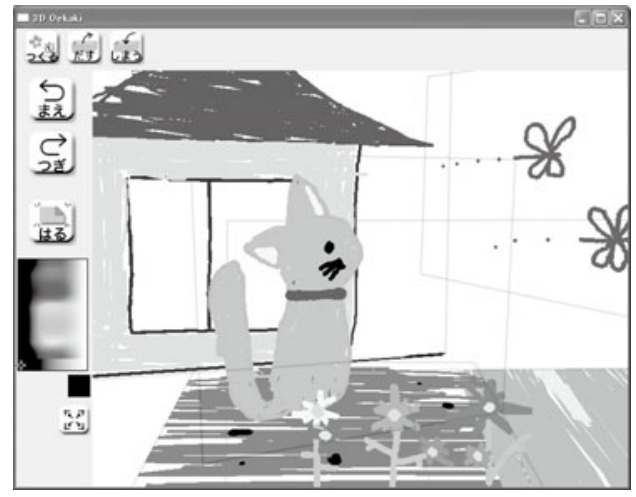

Fig. 3. 3-D paint system

\subsection{Methods of Operation}

Fig.4 shows a layout of the buttons of the 3-D Remote (Wii Remote), and Fig.5 shows a coordination system of the system. The system has functions of translating and rotating papers, and translating and rotating a view by using the 3-D Remote.

1. Translations: A user can translate a paper by hitting the arrow keys of the 3-D Remote. If the 3-D Remote is horizontal, a paper will translate back, forth, right or left on the X-Z plane. If the 3-D Remote is vertical, a paper will translate up, down, right or left on the $\mathrm{X}-\mathrm{Y}$ plane. The threshold value is 45 degrees. 
2. Rotations: A user can rotate a paper by holding the B-button and rotating the 3-D Remote. If a user rotates the 3-D Remote around the X-axis, a paper will rotate around the $\mathrm{X}$-axis. If a user rotates the 3-D Remote around the Z-axis, a paper will rotate around the $\mathrm{Y}$-axis.

3. Changing an object: A user can move a cursor by holding the 3-D Remote in the direction of a display and moving the 3-D Remote. And a user can change an object by pointing to an another object and hitting the A-button.

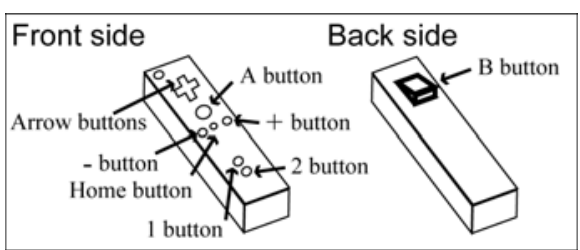

Fig. 4. 3-D Remote

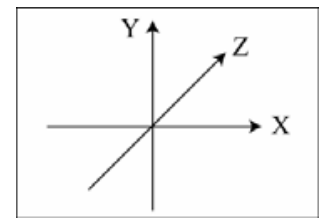

Fig. 5. Coordinate system

\section{Experiment Overview}

We conducted the experimental tests in August 2009, January 2010, and August 2010 in a pre-school as shown Fig.6. The examinees were 14 children 4-years-old, 17 children 5-years-old, and 11 children 6-years-old, 26 boys and 16 girls. 15 children took the experiments twice in the first experiment and also in the second experiment.

We tested Translation-test, Rotation-test, and Paper-lay-out-test for children in every experiment. In August 2010, we used the WPPSI IQ test. These experiments were conducted in individual room. We explained to them the method of operation, and tests before tests. The required time of experiments was about 20 minutes. Fig.7 shows a photo of the experiment. Fig. 8 shows the process of the tests.

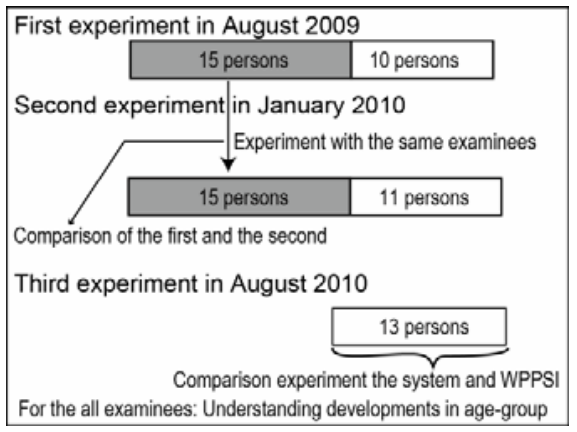

Fig. 6. Experiment plan

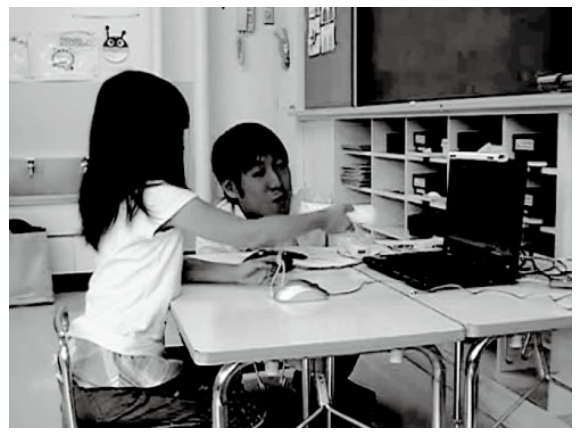

Fig. 7. Experiment in a kindergarten 


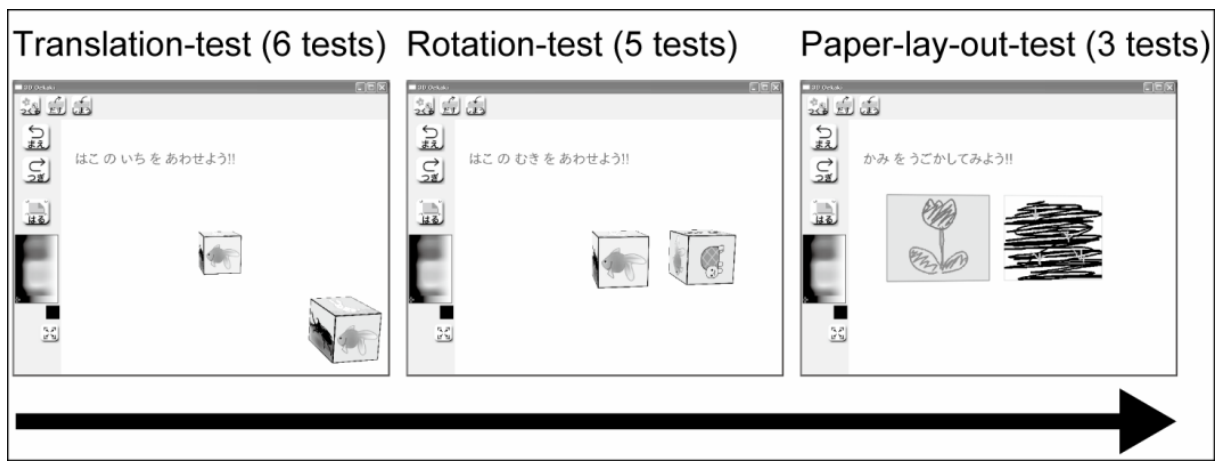

Fig. 8. Process of tests

\section{Difference of Operation Ability in Space in Half a Year}

\subsection{Experiment Methods}

We describe Translation-test and Rotation-test as follows:

- Translation-Test: Two cubes of the same size but different colors are arranged at different positions in advance. An examinee translates the highlighted cube at the center to another cube. There are 6 different tests as shown Fig.9.

- Rotation-test: Two cubes of the same size but different colors are arranged in different directions in advance. An examinee rotates the highlighted cube until the same direction is obtained as the other one. There are 5 different tests as shown in Fig. 10.

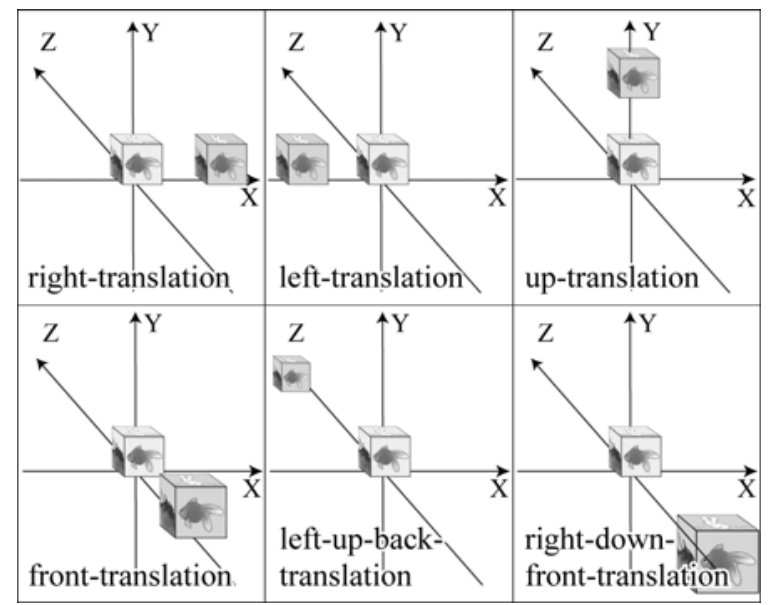

Fig. 9. Translation-test 


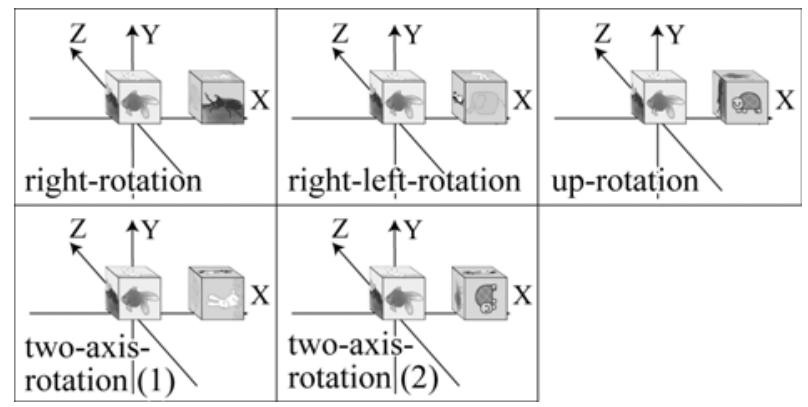

Fig. 10. Rotation-test

\subsection{Experimental Result}

Fig.11 shows the average times and the standard deviations of time required to complete a test, and the rates of completion. In the second experiment, most of the average times of the Translation-test and the Rotation-test are shorter than the first experiment. And in the second experiment, most rates of completion of the Translation-test and the Rotation-test are greater than the first experiment. By t-test, the average times of right-rotation, right-left-rotation, and two-axis-rotation(2) are significantly different between the first experiment and the second experiment $(\mathrm{p}<.05)$. By F-test, the average times of right-translation, up-translation, right-rotation, right-left-rotation, and up-rotation are significantly different between the first experiment and the second experiment. This result provides evidence that shows the difference of children's development of operation ability in space. Moreover, the result provides evidence that children can recognize the virtual space.

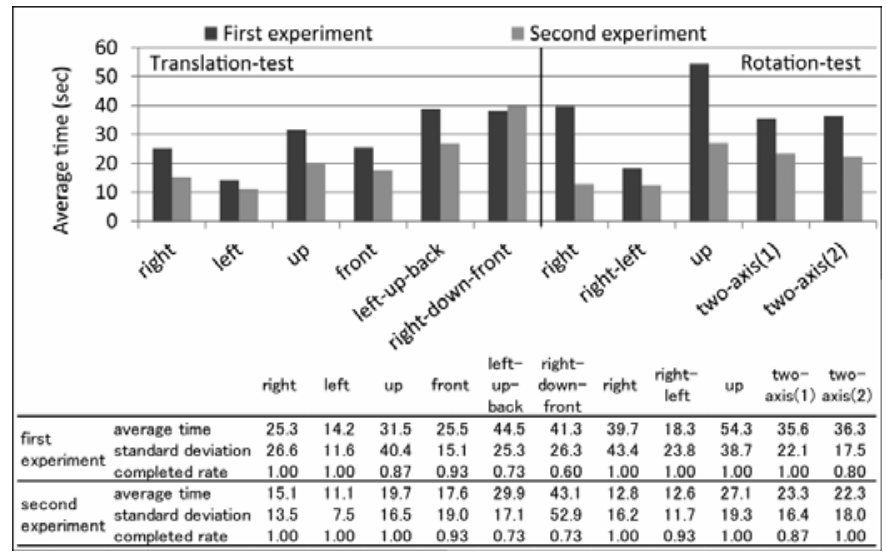

Fig. 11. Average time and standard deviation in 6 month

Fig.12 shows the average times and the rates of completion of all the examinees of the Translation-test and the Rotation-test in their age-group. The average times 
decrease with the rates of completion increasing with advancing age. However, the average time of 5-year-old children for the Translation-test is longer than for the 4year-old children. Because the rates of completion increase from 5-year-old, the examinees of 5-year-old that could complete the tests with longer time increased. This result shows that we can measure development of operation ability in space using the system.

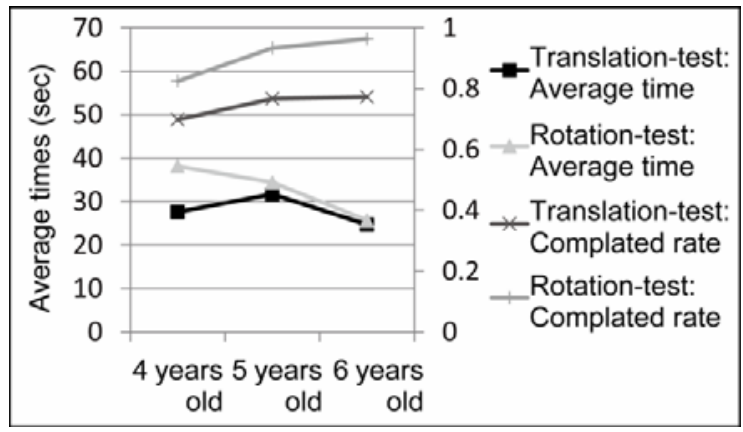

Fig. 12. Average time and rate of completion

\section{Correlation of the System and WPPSI}

\subsection{Experiment Methods}

In our experiment in August 2010, we used this system and the Japanese version of the WPSSI for the 14 children. The aim of this experiment was to validate the evidence that the system is an effective tool to measure a developmental stage of spatial expression ability.

The Japanese version of the WPPSI is an intelligence test designed for children ages 3 years 10 months to 7 years 1 months. The WPPSI provides subtests with 6 verbal tests and 5 performance tests. And it provides Verbal and Performance IQ scores, and a Full Scale IQ score. However, the WPPSI is not specialized in understanding the spatial expression ability. We conducted all 5 performance subtests of Animal House, Picture Completion, Mazes, Geometric Design, and Block Design.

\subsection{Experimental Result}

Fig.13 shows the examples of correlation between the required times of the system and the scores of the WPPSI. The upper graphs show correlations between average times of the Translation-test and the WPPSI, and the lower graphs show correlations between average times of the Rotation-test and the WPPSI.

In the Translation-test and the WPPSI, the average time increases with the score of WPPSI increasing. This result is the same in other all Translation-tests. By test of no correlation, Picture Completion and Geometric Design correlate significantly with the Translation-test $(\mathrm{p}<.05)$. Moreover, the Performance IQ score correlates significantly with the Translation-test. This result shows that children have an ability of 
systematizing a way to reach a goal in Mazes, or an ability of painting by comparing an original with their own picture in Geometric Design effect in virtual space. But, we found no correlation between the Rotation-test and the WPPSI. However, this result shows that the system can analyze abilities that are hard for the WPPSI to measure because the results of the Rotation-test improve with advancing age in section 4.2.

That is, the system obtained an equal result to the WPPSI for analyzing spatial expression ability. And the system can analyze spatial expression abilities that are hard to understand with the WPPSI.
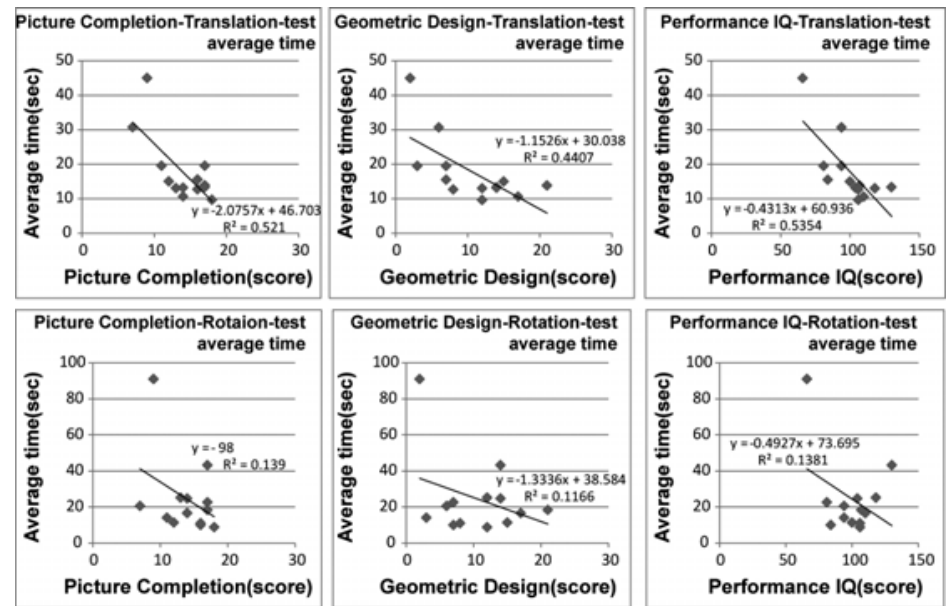

Fig. 13. Correlation between the system and WPPSI

\section{Paper-Lay-Out-Test}

\subsection{Experiment Methods}

Paper-lay-out-test is an experiment to test the ability to express the positional relation between two papers. Two illustrated papers are arranged in virtual 3-D space of the system in advance. In real space, the test examiner shows a relation to the child using the two illustrated papers. And in the virtual 3-D space of the system, the child will arrange the papers similar to the relation.

The Paper-lay-out-test consists of Parallel-lay-out-test, Vertical-lay-out-test(1), and Vertical-lay-out-test(2) as in Fig.14. We added vertical-lay-out-test(2) from January 2010.

\subsection{Experimental Result}

Fig.15 shows the 9 typical expression patterns that we obtained from the examinees. Table.1 shows the frequencies of expression patterns of all examinees and the frequencies of examinees of the first experiment and the second experiment, the underlined data shows the correct expression pattern. 


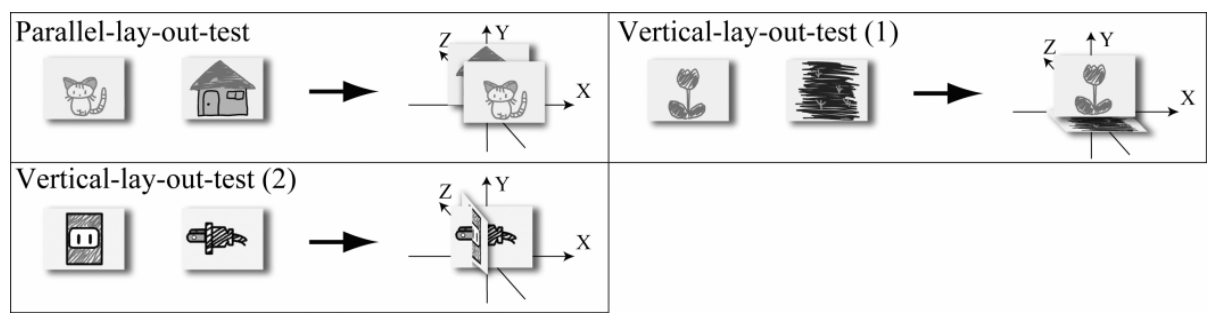

Fig. 14. Paper-lay-out-test

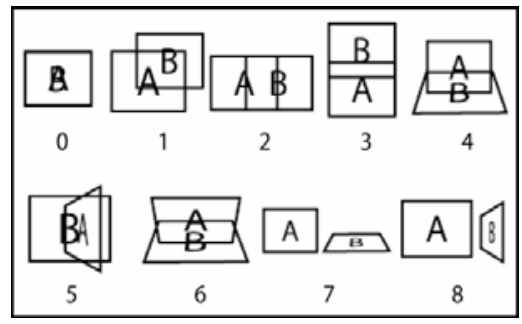

Fig. 15. Expression patterns

Table 1. Result of Paper-lay-out-test

\begin{tabular}{llllllll}
\hline & \multicolumn{3}{l}{ Parallel-lay-out } & \multicolumn{3}{l}{ Vertical-lay-out(1) } & Vertical-lay-out(2) \\
patterns & all & first & second & all & first & second & all \\
0 & 36 & 10 & 8 & 24 & 6 & 4 & 14 \\
1 & $\mathbf{8}$ & $\underline{\mathbf{2}}$ & $\mathbf{1}$ & 9 & 4 & 1 & 1 \\
2 & 5 & 2 & 2 & 1 & 0 & 0 & 5 \\
3 & 1 & 0 & 1 & 3 & 0 & 2 & 0 \\
4 & 0 & 0 & 0 & $\underline{\mathbf{1 4}}$ & $\underline{\mathbf{3}}$ & $\underline{\mathbf{5}}$ & 0 \\
5 & 7 & 1 & 3 & 2 & 0 & 2 & $\underline{\mathbf{1 6}}$ \\
6 & 1 & 0 & 0 & 2 & 1 & 1 & 0 \\
7 & 2 & 0 & 0 & 3 & 1 & 0 & 0 \\
8 & 1 & 0 & 0 & 1 & 0 & 0 & 1 \\
\hline
\end{tabular}

In the pattern 0 , two papers are arranged at the same position, is highest frequency in all the tests except the Vertical-lay-out-test(2). This result is caused by a lack of understanding the experimental descriptions, a confusion of the Translation-test, or undeveloped spatial expression ability.

In the Parallel-lay-out-test, 8 examinees expressed the correct expression for the pattern 1. The rest of children expressed the pattern 2 or 5 . We could not obtain significant differences between the first experiment and the second experiment. This result is caused by the following reasons: it was hard for examinees to understand whether they should arrange the papers with opening a gap or arrange the papers to the same position such as the pattern 0; or undeveloped spatial expression abilities.

In the Vertical-lay-out-test(1), 14 examinees expressed the correct expression for the pattern 4 . We see that the number of the pattern 4 increases, the number of the 
pattern 3 and 5 as nearly correct expression increases, the number of the pattern 0 and 1 decreasing in half a year. In the Vertical-lay-out-test(2), the highest number of 16 examinees expressed the correct expression for the pattern 5.

This result shows that we are able to obtain spatial expression in 3-D space, and the system obtained data showing children's development of the expression abilities of relations with advancing age.

\section{Conclusion}

We prepared a 3-D paint system to understand the developmental stage of spatial expression ability, and conducted evaluation experiments using the system in a preschool. The results are summarized as follows:

- Children are able to recognize virtual 3-D space of the system, and are able to express images in virtual 3-D space.

- The data shows the trend that spatial expression ability in space becomes better with advancing age.

- We obtained correlations between the system and WPPSI. The system can analyze some abilities that are difficult for WPPSI.

In future studies, we will continue to conduct experiments in a pre-school, and make an evaluation method to understand developmental stages of spatial expression ability from the acquired data.

\section{References}

1. Higashiyama, A.: Children's Space Recognition and It's Expression. Ann. Kobe University 83, 71-81 (1989)

2. Wechsler, D.: Wechsler Intelligence Scale for Children-III, San Antonio (1991)

3. Takeuchi, Y.: Can Children Understand Aerial Photographs as Map-like Representations? Ann. Aichi University of Education 6, 43-48 (2003)

4. Ogawa, A.: Young Children's Spatial Perspective Taking Ability: The Relationship between Children's Understanding and Production of Drawings in Two Conditions. Ann. Kyoto University 52, 412-426 (2006)

5. Wechsler, D.: Wechsler Preschool and Primary Scale of Intelligence, San Antonio (1967)

6. Ito, K., Fukuda, T.: Wii Remote. J. Transactions of the Japan Society of Mechanical Engineers 110, 908-909 (2007) 